\title{
Special Issue on Innovative Design of Complex Products
}

\author{
Jian-Rong Tan*
}

With the development of science and technology as well as the diversification and individualization of market demand, modern products update more and more rapidly. The rapid development of new products to satisfy the increasingly improved and personalized customer demands greatly depends on the progress in the innovative design theory and performance optimization method. In this situation, scholars all over the world in the field of mechanical engineering have recently conducted a great deal of research work on the innovative design of products, development of innovative mechanisms and machines, performance analysis and optimization methods for complex products, and so on. Accordingly, significant progress has been made in the field.

Thus, we issue a special issue column in Chinese Journal of Mechanical Engineering (CJME) on Innovative Design of Complex Products. The aim of this special issue is to provide an overview of current research on the new theories and methods for the innovative design and performance optimization of complex products as well as the latest developed innovative mechanisms and machines. As a result of this Call for Papers, over 30 fulllength papers were submitted, which passed all rounds of anonymous review processes typical for the Chinese Journal of Mechanical Engineering. Twelve papers were accepted and included in this special issue.

Product innovation is the creation of new concepts to plan and realize technological and functional details in the product to satisfy market and customer demands, which needs a cognitive design method based on the demands of various customers for the production personalization. Although open-architecture product (OAP) can improve the production personalization and lead to the massive production innovation, there is still a lack of effective methods for developing OAPs. To

\footnotetext{
*Correspondence: egi@zju.edu.cn

State Key Laboratory of Fluid Power and Mechatronic Systems, Zhejiang University, Hangzhou 310027, China
}

overcome this difficulty, Peng et al. introduce an adaptable design method for OAPs to meet the product personalization, which provides solutions for planning modules and implementation of OAPs. Jia et al. propose a patentable ideation framework for achieving more patentable ideas in analogy-based design and suggest three heuristics for searching the set of candidate target systems with similar design problem where the source design can be transferred.

Also, great efforts have been made to develop new mechanisms and machines in recent years. For example, Yang et al. develop a kind of triangular prism mast (TPM) with tape-spring hyperelastic (TSH) hinges and establish the theoretical model of stretching and compression stiffness. They also investigate the influence of thickness, section angle and radius based on TPM finite element model and parameter study, and verify the precision of theoreti$\mathrm{cal}$ and finite element models. Qiao et al. develop a lightweight, less driven under-actuated robotic hand to realize on-orbit capture in space and propose a new analysis method for configuring the number of knuckles of finger by taking the maximum grasping space into account. He et al. conduct a comparative study on a group of flexure mechanisms with different degree of symmetry (DoS), which are synthesized based on freedom and constraint topology method. Sun et al. propose a unified and systematic approach for the topology design and kinematic analysis of parallel kinematic machines (PKMs) based on finite and instantaneous screw theory, which enables the implementation of analytical description, formulation and operation for both finite and instantaneous motions by the same mathematical tool and can be utilized in the innovative design of different kinds of PKMs.

For the purpose of ensuring the performance and precision of newly developed products, we often need to analyze their static and dynamic characteristics in design phase. For instance, Zhou et al. present a complete design process of vehicle powertrain mounting system including the vibration decoupling, vibration simulation analysis,
Springer Open

(c) The Author(s) 2018. This article is distributed under the terms of the Creative Commons Attribution 4.0 International License (http://creativecommons.org/licenses/by/4.0/), which permits unrestricted use, distribution, and reproduction in any medium, provided you give appropriate credit to the original author(s) and the source, provide a link to the Creative Commons license, and indicate if changes were made. 
topology optimization and experimental verification in order to improve the vibration isolation performance and dynamic characteristics of the mounting system. Duan et al. numerically investigate the flow-induced vibration of planar elastic tube bundle based on a twoway fluid-structure interaction calculation, and draw the conclusion that the vibration of out-of-plane is the main vibration form with the typically sinusoidal behavior and that the vibration balance position and amplitude are susceptible to the fluid velocity.

Manufacturing and assembling errors are indispensible in engineering practice, which need to be considered and analyzed to ensure the performance and precision of products. Liu et al. propose an error equivalent model of revolute joints considering both radial and axial clearances for investigating the pointing accuracy of the antenna pointing mechanisms. Yuan et al. develop a precisely generalized finite element model of helical gear system to investigate the influences of manufacturing errors including short term and long term components on the quasi-static and dynamic behaviors of the system, and conclude that both of these manufacturing errors have notable influence on mesh stiffness, loaded static transmission error and loaded composite mesh error of helical gears.

Structural optimization approaches aimed at improving the mechanical performance of complex products and reducing manufacturing cost are also proposed. For example, Ma et al. put forward a light-weight design method for the force-performance-structure of complex parts based on collaborative optimization of structural topology and size. Cheng et al. propose a new method for evaluating the feasibility and objective robustness of uncertain structures based on the new concept of interval violation vector, and realize the robust optimization of uncertain structures with interval parameters based on Kriging technique and nested genetic algorithm.

In summary, this issue attempts to provide the latest research achievements about the innovative design of complex products, including the general theories and methods for innovative design, innovative development and mechanical analysis of complex products, performance analysis and optimization considering indispensible errors and so on. All of these research achievements promote the progress of mechanical engineering and the emergence of new products. The Guest Editor greatly appreciates the authors' excellent contributions to this special issue and the anonymous reviewers' suggestions on the papers.

\section{Guest Editor}

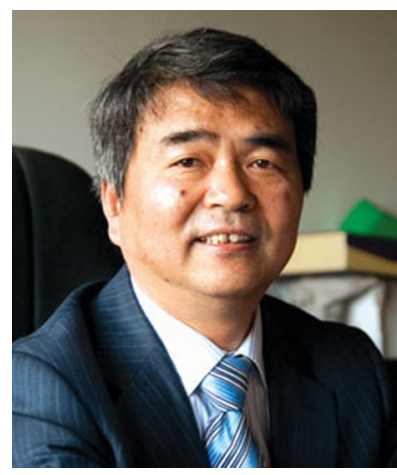

Jian-Rong Tan is an Academician of Chinese Academy of Engineering and a Professor of Mechanical Engineering at Zhejiang University, China. He is the Vice Chairman of Chinese Mechanical Engineering Society and a member of the editorial board of Chinese Journal of Mechanical Engineering. He received his $\mathrm{PhD}$ degree in Applied Mathematics from Zhejiang University, China, in 1992. His current research interests include theory of mechanical design, digital design and manufacturing, and CAD\&CG. He has published 8 books and authored or coauthored over 142 papers. He has received the 2nd prize of National Award for Science and Technology Progress of China four times and awarded the 1st prize of Zhejiang Province (China) seven times.

\section{Authors' Contributions}

The author read and approved the final manuscript.

Ethics approval and consent to participate Not applicable.

\section{Publisher's Note}

Springer Nature remains neutral with regard to jurisdictional claims in published maps and institutional affiliations.

Received: 16 April 2018 Accepted: 16 April 2018

Published online: 06 May 2018

\section{Submit your manuscript to a SpringerOpen ${ }^{\circ}$ journal and benefit from:}

- Convenient online submission

- Rigorous peer review

- Open access: articles freely available online

- High visibility within the field

- Retaining the copyright to your article

Submit your next manuscript at springeropen.com 